\title{
Design of an Automatic Defect Identification Method Based ECPT for Pneumatic Pressure Equipment
}

\author{
Bo Zhang, ${ }^{1}$ YuHua Cheng $\left(\mathbb{D},{ }^{1}\right.$ Chun Yin $\mathbb{D}^{1},{ }^{1}$ Xuegang Huang $\mathbb{D}{ }^{2}$ \\ Sara Dadras, ${ }^{3}$ and Hadi Malek (iD ${ }^{3}$ \\ ${ }^{1}$ School of Automation Engineering, University of Electronic Science and Technology of China, Chengdu, 611731, China \\ ${ }^{2}$ Hypervelocity Aerodynamics Institute, China Aerodynamics Research \& Development Center, Mianyang 621000, China \\ ${ }^{3}$ Electrical and Computer Engineering Department, Utah State University, Logan, UT 84321, USA \\ Correspondence should be addressed to YuHua Cheng; yhcheng@uestc.edu.cn
}

Received 4 July 2018; Revised 31 August 2018; Accepted 25 September 2018; Published 22 October 2018

Academic Editor: Lingzhong Guo

Copyright (C) 2018 Bo Zhang et al. This is an open access article distributed under the Creative Commons Attribution License, which permits unrestricted use, distribution, and reproduction in any medium, provided the original work is properly cited.

In this paper, in order to achieve automatic defect identification for pneumatic pressure equipment, an improved feature extraction algorithm eddy current pulsed thermography (ECPT) is presented. The presented feature extraction algorithm contains four elements: data block selection; variable step search; relation value classification; and between-class distance decision function. The data block selection and variable step search are integrated to decrease the redundant computations in the automatic defect identification. The goal of the classification and between-class distance calculation is to select the typical features of thermographic sequence. The main image information can be extracted by the method precisely and efficiently. Experimental results are provided to demonstrate the capabilities and benefits (i.e., reducing the processing time) of the proposed algorithm in automatic defect identification.

\section{Introduction}

As one of the most significant energy storage and transmission kind of equipment, the pneumatic pressure equipment has been widely used in modern aerospace industries, such as manned spacecraft and ground wind tunnel testing in Figure 1. Moreover, given its wide range of applications, the equipment possesses a variety of specifications that make it flexible. The flexibility of their specifications adds complexity to problem of inspecting and testing this important equipment to identify defects. Researchers have studied methods to characterize pressure vessel properties in recent years. J. Lee et al. proposed a method of estimating the design pressure in a prismatic storage vessel for liquefied natural gas [1]. H. AlGahtaniet et al. studied the local pressure testing of spherical vessels with nozzles [2]. J. Proczka et al. reported guidelines for the efficient design and sizing of Small-Scale Compressed Air Energy Storage (SS-CAES) pressure vessels [3]. P. Blanc studied the residual burst pressure of composite cylinders after mechanical impacts on their cylindrical part [4].
These studies, however, do not explore the impact of defects related to the on-going use of pressure vessels made of diverse materials in a wide range of operating environments.

The complicated usage environments and diversity of materials cause the pneumatic pressure equipment to generate fatigue cracks, corrosion pits, and other defects. Therefore it is important to detect the potential defects for the pressure equipment. Nondestructive testing and evaluation (NDT\&E) [5-10] are important to ensure the safety of manufacturing environments and equipment operation. K. Schabowicz et al. presented some nondestructive methods for the testing of concrete members and fibre cement boards [11]. Furthermore, the criteria for special specimen inspection are distinct, so various detection methods are needed including surface nondestructive testing, appearance inspection, and physicochemical test. The surface nondestructive testing mainly includes magnetic particle testing and penetrant testing. Due to the advantages of surface nondestructive testing, like high sensitivity and easy identification, this method been broadly adopted. However, there are many problems that 


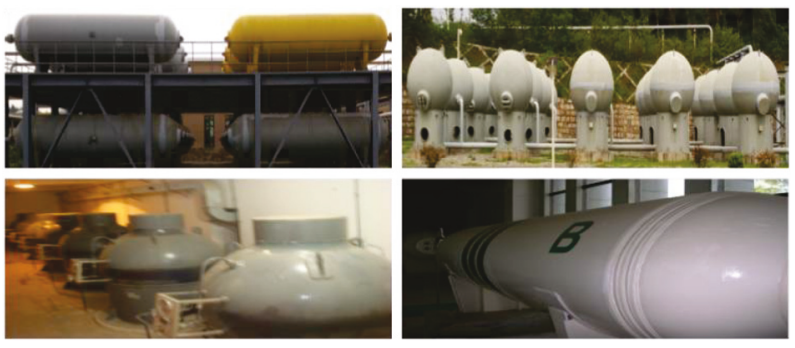

FIGURE 1: Pneumatic pressure equipment.

need to be resolved in its practical application, like high labor intensity, low efficiency, pollution of the environment, poor safety, and so on. In recent years, ECPT has been developing rapidly. In addition to not damaging the body, the method is fast, is efficient, and can solve the issues of regular methods. So in this paper, the eddy current pulsed thermography is implemented in the detection of defects of pneumatic pressure equipment. Researchers also have made valuable contributions in processing data [12-15], which can be applied in (NDT\&E). P. Zhu et al. presented an improved feature extraction algorithm for automatic defect identification based on eddy current pulsed thermography [16]. H. Yu utilized the normalized fuzzy weighting functions to dispose the records of experiment [17].

Nevertheless, the above-mentioned researches possess low efficiency and limitations in processing data from lowalloy steel material. Besides, there are many methods, such as Independent Component Analysis (ICA); the processed feature information will be distorted after restructuring, because of data normalization, lending to no visual effect in detection. In recent years, adaptive technique has gained a lot of interest to enhance the efficiency of processing data [18-21]. To make the detection system be more useful for detecting the defect, it may own the ability of adapting property. A novel characteristic identification algorithm that uses the similarity of the typical transient thermal responses (TTRs) with the mixing vectors (the vectors of the pseudo-inverse matrix of the demixing matrix) in ICA is proposed. It solves the poor accuracy and low efficiency of the ICA efficiently by using the choice of a known message. The proposed algorithm has four steps. First the thermal imager collects the thermal image sequence of the pneumatic pressure equipment. Second, the TTRs are separated into parts using threshold values with a variable interval search. The variable interval search in an infrared image sequence can reduce the repetitive computation and hold the typical feature of the pneumatic pressure equipment. Third, the membership matrix and the clustering center are calculated to classify the acquired TTRs. Moreover, the rule of the largest distance of two class is used to identify the typical TTRs. Fourth, the typical TTRs are transformed into a two-dimension matrix in linear time. The main features of the infrared image sequence in the pneumatic pressure equipment can be extracted by the typical TTRs. Experimental results indicate the algorithm can select the typical feature more precise than ones of the ICA. Furthermore, the processing time of the presented algorithm is shorter than ones of the ICA. Therefore, the new algorithm

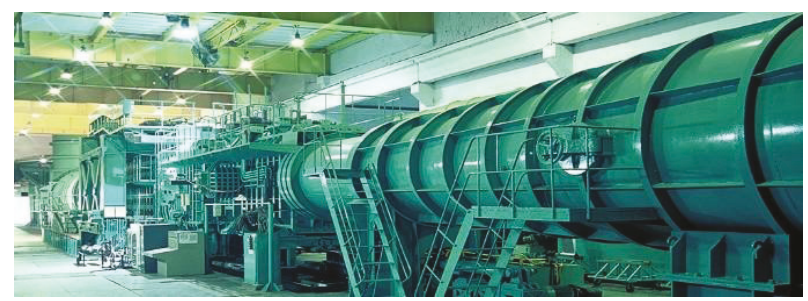

Figure 2: Pneumatic pressure equipment in China Aerodynamics Research \& Development Center.

can extract the main image information of the pneumatic pressure equipment accurately and efficiently in comparison to the ICA.

\section{Background: Pneumatic Pressure Equipment and Nondestructive Testing}

2.1. Pneumatic Pressure Equipment. The manufacture of high pressure vessels is complex, involving the coordination of subject knowledge and professional technology in many industries, including metallurgy, corrosion and protection, mechanical processing, safety protection, chemical engineering, and testing. The recent advances in technologies across various industries, including nondestructive testing, have enabled considerable progress in pressure vessel manufacturing.

(1) Varieties and specifications: the types of material can be mainly divided into carbon steel, low-alloy steel, and a small amount of stainless steel. Moreover, the main specification of external diameter has dozens of kinds.

(2) Complex usage environment: pressure vessels and pipes often suffer from high pressure, some special aerodynamic erosion, and environmental corrosion in the open air, which usually lead to cracks, perforation, fatigue damage, and corrosion of the equipment.

(3) Devastating consequences of defects: accidents not only endanger the safety of personnel, equipment, and the plant, but also lead to delay of test task, failures, and so on.

2.2. Nondestructive Testing. Surface nondestructive testing is the key test method of pressure equipment, such as pressure piping and pressure vessels. Surface nondestructive testing basically includes magnetic particle testing and penetrant testing. The method possesses numerous advantages such 
as high defect detection rate and high sensitivity. But there are also many problems, including high labor intensity, long overhaul cycle, low efficiency, pollution of the environment, and poor safety. So there exist a great many obstructors for development of detection skill. In flammable explosive environment, because of the safety risks, the traditional types of surface nondestructive testing methods (magnetic particle, penetrant) cannot be implemented in the field.

In recent years, surface detection by eddy current has been (developing) rapid. In addition to not damaging the body, the method is fast and efficient and can achieve large area rapid detection reducing manpower and material resources. The surface detection by eddy current is a method that utilizes thermal infrared image analysis.

The temperature field distribution is based on a vortex phenomenon that can be measured using infrared detection. The device is a thermal infrared imager with high speed and high resolution. Moreover, through the analysis of the infrared thermal image sequence, we could detect the changes of electromagnetic and thermal characteristics of the structure and material defects. It can detect the surface and near surface by using the eddy current effect; it also can detect deeper defects. In the light of Joule's law, the eddy current is turned into Joule heat in the testing piece generating high temperature and low temperature zones. The infrared thermal imager collects these temperature changes in the specimen. The collected data are analyzed to compute anticorrelation results and detect the presence of defects.

Eddy density can be indicated as $J_{e}=\sigma \times(\partial A / \partial t)$, in which $A$ expresses the magnetic vector bit and $\sigma$ indicates the electrical conductivity of materials. In the depth direction, the eddy current density is $J_{e}(z)=J_{e}(0) \cdot e^{-z \sqrt{\pi \mu \sigma f}}$, in which $z$ is the depth, $\mu$ is the permeability of testing material, and $f$ is the frequency of alternating current flowing through an exciting coil. The depth of eddy density has an attenuation to the $1 / e$ of the strength surface, which is called as skin depth $\delta=1 / \sqrt{\pi \mu \sigma f}$. According to Joule's law, the heat power is $P_{w}=(1 / \sigma)\left|J_{e}\right|^{2}=(1 / \sigma)|\sigma E|^{2}$ and $\mathrm{E}$ is electric field intensity. The Joule heat $\mathrm{Q}$ is transmitted inside the material, and the heat propagation equation is $\rho C_{p}(\partial T / \partial t)-\Delta\left(\sigma_{T} \nabla T\right)=Q$, in which $\rho$ is material density, $C_{p}$ is specific heat capacity of materials, $\sigma_{T}$ is thermal conductivity of materials, and $T$ is temperature of materials. The crack depth is larger than eddy current skin depth in this specimen, so

$$
J_{e}=\sqrt{2} \beta H \frac{\sqrt{\cosh (2 \beta x)-\cos (2 \beta x)}}{\sqrt{\cosh (\beta b)+\cos (\beta b)}},
$$

in which $b$ is height, $\mathrm{H}$ is magnetic field intensity, and

$$
\beta=\sqrt{\pi f \sigma \mu}=\frac{1}{\delta} .
$$

The thermal power in the unit volume of the two sides of the crack is $P_{w}=0.5(1 / \sigma) \int J_{e}^{2} d x$, when the eddy skin depth is smaller than the plate thickness $(\delta \ll b)$, the thermal power $P_{w}=\beta H^{2} /(2 \sigma)$. Recognizing the defects change the heat distribution in the parts, or zones of a specimen, the principal component analysis enhances the zones where the thermal response and the eigenvector has similar trends. So, in the enhancement of defects in a principal component, the amplitude of other regions will be inhibited and distributed in a very small range. In this paper, a new algorithm in view of principal component selection is presented to automatically extract the principal components containing defects.

\section{Introduction of Proposed Algorithm in ECPT}

First of all, some definitions are introduced in the Appendix to make the algorithm clear. Then, the proposed algorithm is explicit as follows.

Step 1. 3D matrix $D$ saves the initial image sequence. Every pixels value of thermal images is saved in the matrix $D$. The third-dimension of $D$ is the time axis $t$.

Step 1.1. Search for $D\left(R_{m m}, C_{m m}, T_{m m}\right)$. To find the length of the interval in the column coordinate, this step sets $(K=$ $1,2, \cdots k \cdots)$ temperature thresholds $T(m),(m=1,2, \cdots K)$ from large to small; moreover the row which includes the highest temperature point $D\left(R_{m m}, C_{m m}, T_{m m}\right)$ is divided into $K+1$ parts.

Step 1.2. Set temperature thresholds $T_{-} C L^{k}$, for each part, and the highest temperature point $D^{k}\left(R_{m m}, C^{k},:\right),(k=$ $1,2, \cdots, K)$ will be found in every part.

Step 1.3. Set $P(P=1,2, \cdots p \cdots)$ temperature thresholds $T(n),(n=1,2, \cdots P)$ from large to small, at the same time, the column which includes the highest temperature point $D\left(R_{m m}, C_{m m}, T_{m m}\right)$ is divided into $P+1$ parts. After the row is divided, the temperature value of the column also should be cut apart rationally in order to extract the characteristics of the defect more accurately.

Step 1.4. Set temperature thresholds $T_{-} R L^{P}$, for each part, and the highest temperature point $D^{p}\left(R_{m m}, C^{p},:\right),(p=$ $1,2, \cdots, P)$, will be found in every part. The number of each block is regarded as $l e n_{p},(p=1,2, \cdots)$. Moreover, the same method as that of column is employed to find the row variable interval $R L^{p}$. The specific procedure of searching for interval is in the following.

(a) Set $(K=1,2, \cdots k \cdots)$ temperature thresholds to divide the TTR into $K+1$ parts.

(b) Set temperature thresholds $T_{-} C L^{k}$ for each part.

(c) Search for the highest temperature point $D^{k}\left(R_{m m}, C^{k}\right.$, :). $c,:))$

(d) Calculate the PCC of $\left(D^{k}\left(R_{m m}, C^{k},:\right)\right.$ with $D^{k}\left(R_{m m}\right.$,

(e) until their PCC is less than $T_{-} C L^{k}$.

(f) The number of $\left(D^{k}\left(R_{m m}, C^{k},:\right)\right.$ whose PCCs with $\left.D^{k}\left(R_{m m}, c,:\right)\right)$ are more than $T_{-} C L^{k}$ is regarded as $C L^{k}$.

(g) Set $(P=1,2, \cdots p \cdots)$ temperature thresholds to divide the TTR into $P+1$ parts.

(h) The same process as the searching for column interval to find the row interval. 
(i) The row interval is regarded as $R L^{p}$.

Step 1.5. What is more, set the threshold DD, initialize $p=$ $1, n=1, z=1$, and carry out the following procedures:

(a) Set $r=1$, save the $D_{k}^{p}(r, c,:)$ into the $X(z,:), r=r+R L^{p}$

(b) If $r \leq l e n_{p}$, Compute the PCC of $D_{k}^{p}(r, c,:)$ with $X(z,:)$, else go to step $(\mathrm{d})$

(c) If $P C C<D D, z=z+1, X(z,:)=D_{k}^{p}(r, c,:), r=$ $r+R L^{k}$, return step (b), else $r=r+R L^{k}$, back to step (b)

(d) If $r<M, p=p+1, z=z+1$ back to step (a), else $c=c+C L^{k}$

(e) If $c<l e n_{k}$, back to step (a); else go to step (f)

(f) If $c<N, k=k+1, c=1$, back to step (a), else the steps are finished

Pneumatic pressure equipment includes high, medium, and low pressure storage containers and inlet/outlet gas pipelines, as shown in Figure 2. The pneumatic pressure equipment stores and conveys compressed air. The main features of these pressure vessels and the significance of their defect inspection are reflected in the following aspects.

The specific calculation process is shown as Figure 3.

Remark 1. For the interval $C L^{k}$, the suitable method to seek out is searching for the length of region with largest temperature variation. What is more, the TTR with largest peak value is always contained in the region with the largest temperature variation. As analysis of Step 1, the length of region with largest temperature variation can be found by the coordinate value of $T_{C L}$. For $R L^{p}$, the homologous setting rule is analogous to $C L^{k}$. The proposed algorithm can include the total typical temperature variations. And the representative temperature variations express the feature in the corresponding image sequence. characteristic pick-up algorithm is put forward to handle data. In the proposed algorithm, the appropriate interval values of column and row $C I^{k},(k=1,2, \cdots K), R I^{p},(p=1,2, \cdots P)$ are set to reduce the repeated calculation. Moreover, the variable interval also can save the significant features. At the same time the proposed algorithm possesses less redundant computation than ICA.

Step 2. Divide $X(:, z)$ into $L$ parts. The specific calculation process is shown as Figure 4.

(a) Set the cluster number L. And, at the same time, initialize the cluster center $m_{o}$; moreover set the number of iterations $c$, and set the weighting coefficient $b$; finally set terminating iterative threshold $\varepsilon$.

(b) Utilize formula to calculate membership function $u_{j}\left(x_{i}\right)=d_{j i}^{-2 /(b-1)} / \sum_{s=1}^{L} d_{s i}^{-2 /(b-1)}$, in which $i=1,2, \cdots, g$, $d_{j k}=\left\|X_{k}-m_{j}\right\|, j=1,2, \cdots, L ; d_{j i}$ expresses the Euclidean distance between the $i^{\text {th }}$ information weight value and the $j^{\text {th }}$ cluster center. $X_{k}$ is the information weight value. $b$ is weighting coefficient. $u_{j}\left(x_{i}\right)$ indicates the degree of the information weight value $x_{i}$ attached to the $j^{\text {th }}$ part.

(c) Update cluster center $m_{j}=\sum_{i=1}^{g}\left[u_{j}\left(x_{i}\right)\right]^{b} x_{i} /$ $\sum_{i=1}^{g}\left[u_{j}\left(x_{i}\right)\right]^{b}$, in which $m_{j}$ presents the $j^{t h}$ cluster center.

(d) Judge whether the absolute value of the objective function difference is smaller than the threshold value. If
$\left\|J_{f}(c)-J_{f}(c-1)\right\| \geq \varepsilon$, and $i<g, i=i+1$, go back to step (b). If $i>g$ and $j<L, j=j+1$, return to step (b). If $j \geq L$, stop. If $\left\|J_{f}(c)-J_{f}(c-1)\right\|<\varepsilon$, stop too.

(e) The membership maximization rule is used to deblur all characteristic information weight and get the category of each information weight. $M_{k}=\arg \max \left(u_{j}\left(x_{i}\right)\right)$.

Remark 2. Making use of the method of computing membership function and updating the clustering center in algorithm can extract feature information accurately and effectively. Taking advantage of the COV to classify data is called hard division. Hard division divides every object into a certain category strictly, and each class is unrelated to each other. However, the actual defect information objective exists intermediation in form and category. Moreover there is no definite boundary conditions to distinguish the class. Therefore, classifying the categories according to the membership and cluster centers of each class can be more accurate. The feature information weights in each category represent the optimal weight of the feature information, making the extracted features more accurate and more reliable.

Step 3. The mean value of the $i^{\text {th }}$ classification is $M E A N^{i}=$ $(1 / R(i)) \sum_{j=1,2, \cdots, R(i)} C L A S_{j}^{i}, i=1,2, \cdots, L$, in which $R(i)$ presents the total number of TTRs in the $i^{\text {th }}$ classification. $N P C C_{j^{t}}^{i},(j=1,2, \cdots, R(t))$ is the noncorrelation value of $M E A N^{i}$ and $C L A S_{j}^{t}, t=1,2, \cdots, L$ and $i=1,2, \cdots, t-1, t+$ $1, \cdots, L$ (the $j^{\text {th }}$ TTR does not belong to the $i^{\text {th }}$ classification). Let $D I S_{j^{t}}^{i}=\left\|N P C C_{j^{t}}^{i}\right\|_{p}$, where DIS $S_{j^{t}}^{i}$ expresses the distance of two classes of TTRs $j$ in the $t^{\text {th }}$ classification with other classes. In $t^{t h}(t=1,2, \cdots, L)$ classification, $F T^{t}=$ $\left\|D I S^{i}\right\|_{\infty}$, in which $F T^{t}$ is the final representation TTR of $t^{\text {th }}$ classification. Save $F T^{t},(t=1,2, \cdots, L)$ into $Y$ (i.e., $Y(:, t), t=$ $1,2, \cdots, L$ saves $\left.F T^{t}, t=1,2, \cdots, L\right)$. The specific calculation process is shown in Figure 5.

Remark 3. The noncorrelation value of $M E A N^{i}$ and $C L A S_{j}^{t}$, $t=1,2, \cdots, L$ is expressed as $N P C C_{j^{t}}^{i}=1-\operatorname{PCC}\left(M E A N^{i}\right.$, $\left.C L A S_{j}^{t}\right)$.

Remark 4. $\|*\|_{p}$ is P-norm, $\|*\|_{\infty}$ is infinity-norm, and $\|*\|$ : $X \longrightarrow R$ satisfies the following:

(1) positivity: $\|x\| \geq 0$, and $\|x\|=0 \Longleftrightarrow x=0$ (2) positive homogeneity: $\|c x\|=|c|\|x\|(3)$ trigonometric inequality: $\| x+$ $y\|\leq\| x\|+\| y\|\| x \|_{p}=\left(\left|x_{1}\right|^{p}+\left|x_{1}\right|^{p}+\cdots+\left|x_{1}\right|^{p}\right)^{1 / p}$, and $\|x\|_{\infty}=\max \left(\left|x_{1}\right|,\left|x_{2}\right|, \cdots,\left|x_{n}\right|\right)$.

Remark 5. The purpose of the proposed algorithm in Step 1 to Step 3 is to select the typical thermal responses. These typical responses have less intimately connection with each other in ECPT. The noncorrelation is larger and the thermal responses are more representative. Moreover, the selected accurate data is classified by Steps 2 and 3; the cluster center and membership function are carried out to classify the defect feature. The classification simplifies the data of image processing and makes the postprocessing more precise. 


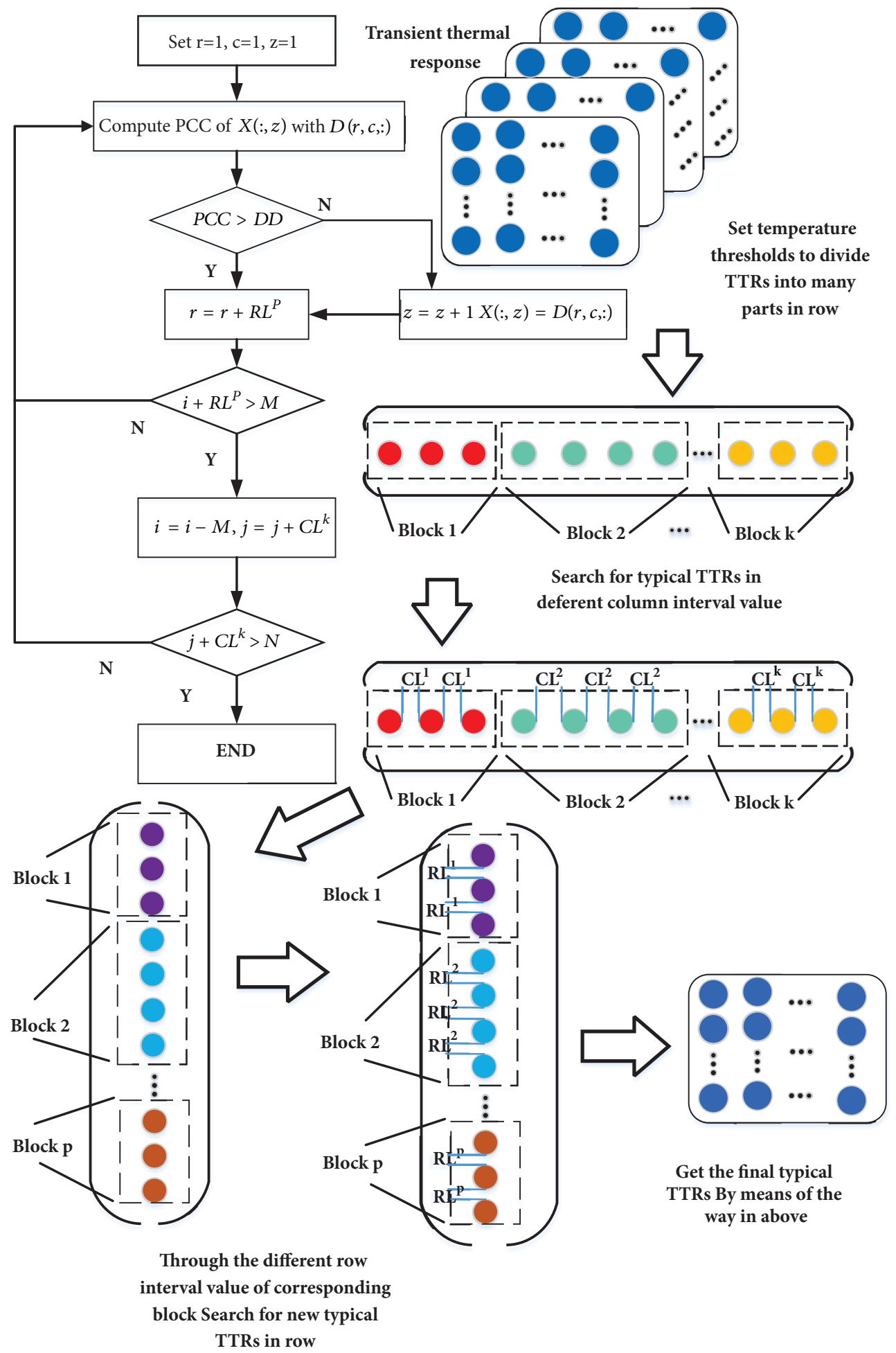

Figure 3: The specific process of Step 1.

Step 4. The $3 d$ initial image sequence matrix $D$ will be transformed into $2 d$ matrix $U$. The elements in one row of $U$ are taken columnwise from $D(:,:, p), p=1,2, \cdots, P$. Calculate $\widehat{Y}$ and solve this linear transformation $S=\widehat{Y} * U$, in which $\widehat{Y}$ expresses the pseudo-inverse matrix of $Y$ and $R$ represents the result of the proposed algorithm. It includes the features of the initial image sequence processed by new algorithm. Moreover, continue to utilize the following way to achieve the extraction of defect feature $O=(1 / N) \sum_{(x, y) \in S} f(x, y)$ in which $O$ expresses the mean of sum of the pixel values, 

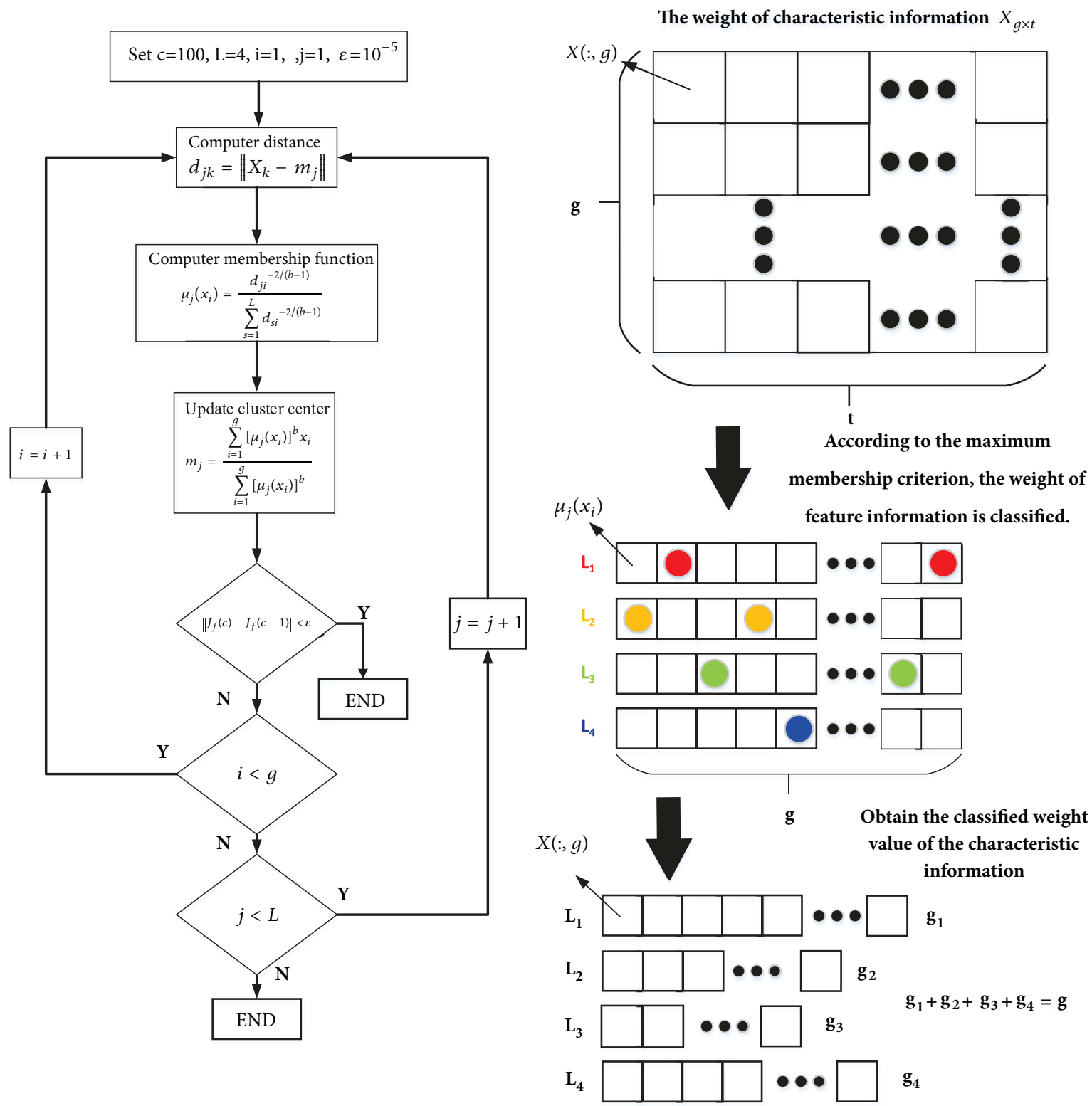

FIgURE 4: The specific process of Step 2.

$\mathrm{f}(\mathrm{x}, \mathrm{y})$ is the pixel values, and $N$ is the number of pixel values. Then we can obtain the uniform measurement of $S$; i.e., $\max |f(x, y)-m|_{(x, y) \in S}<V$, in which $V$ is a threshold. Finally, we can pick up the defect feature from the image.

Remark 6. The final purpose of the proposed algorithm is to extract the defect feature and ignore redundant information. The mean value of selected region is obtained by the similarity criterion of the method. Moreover the calculation of the uniform measurement divides the region into defect feature and other areas.

Remark 7. In order to highlight the efficiency and reasonability of the proposed method, the comparison of the ICA and the proposed algorithm is shown in Figure 6. In the first part "Data whitening", the data in the ICA is dealt with by normalization and then is computed to obtain the whitening vectors according to the corresponding feature vectors and feature matrix. These processes of calculation take a lot of time to dispose data and reduce efficiency of processing. However, the new algorithm does not have these computational procedures, to avoid the redundancy calculation. Hence, the amount of data in the proposed algorithm after processing of pretreatment and whitening is much less than that in the ICA, so, in the postprocessing, the proposed algorithm avoids redundancy calculation and possesses higher efficiency than the ICA. 2) In the second part "Data analysis and comparison", compared with the extracted defect feature information graph, the amplitude of the graph extracted by the new algorithm contains the 


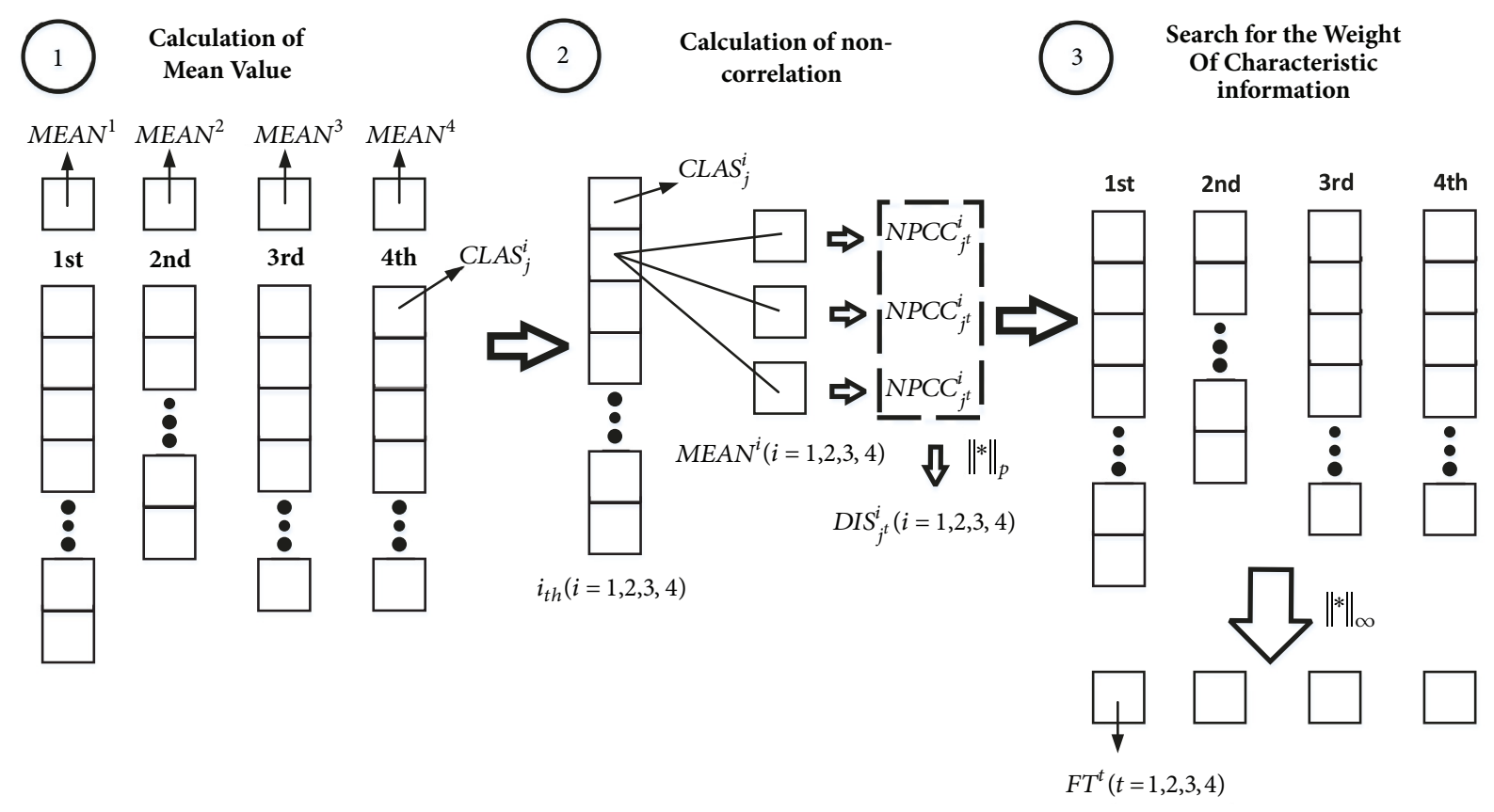

FIgURE 5: The specific process of Step 3.
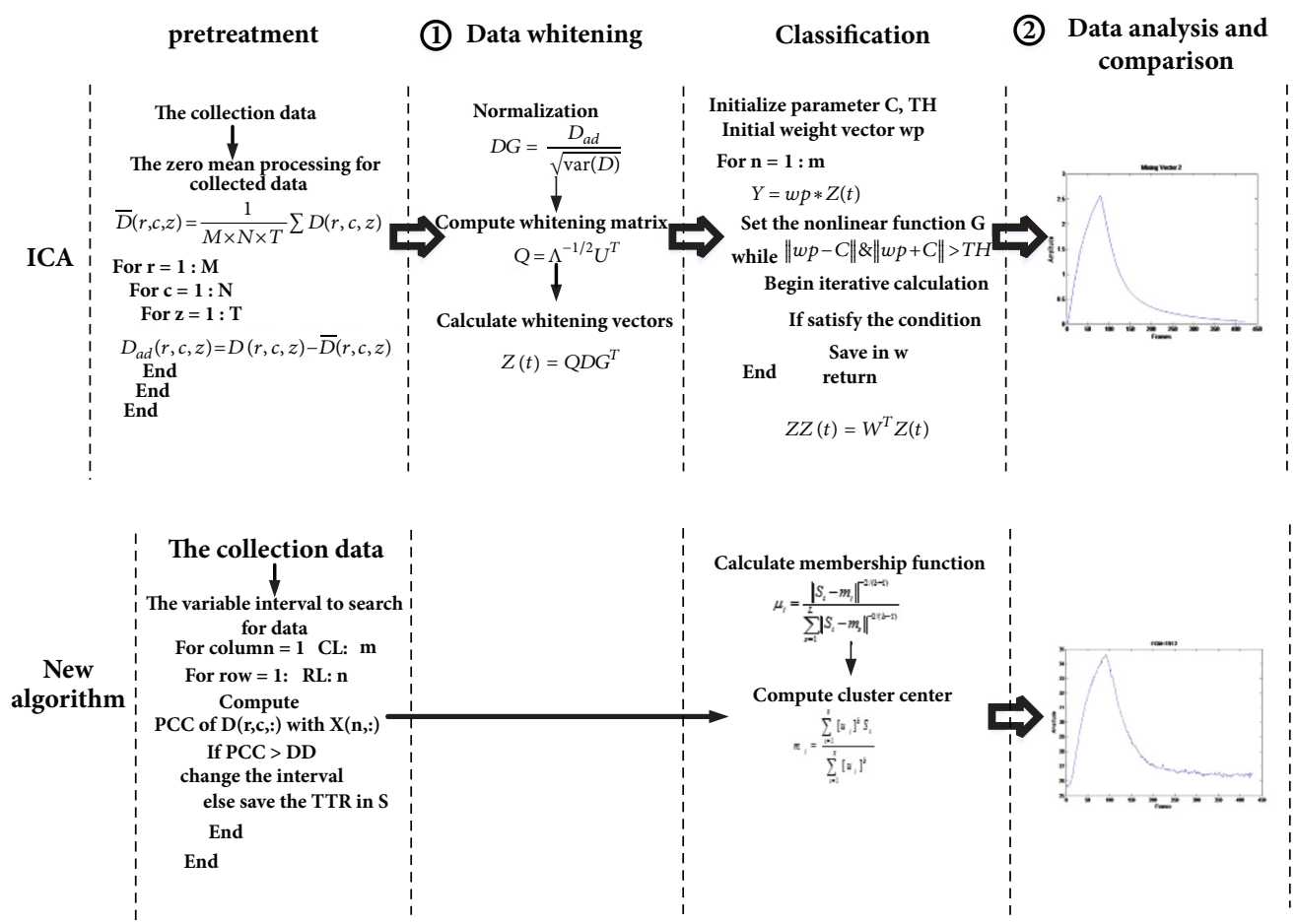

FIGURE 6: The comparison between the proposed method and ICA. 


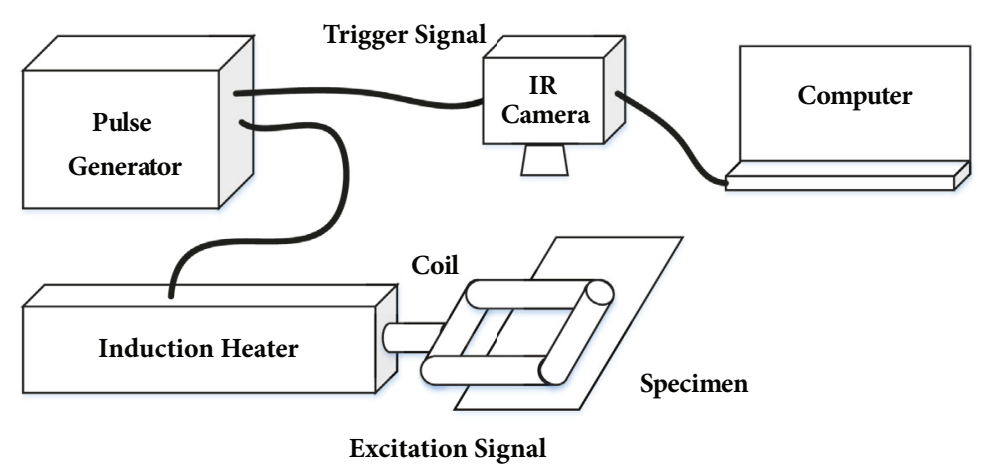

FIGURE 7: The experimental schematic diagram.

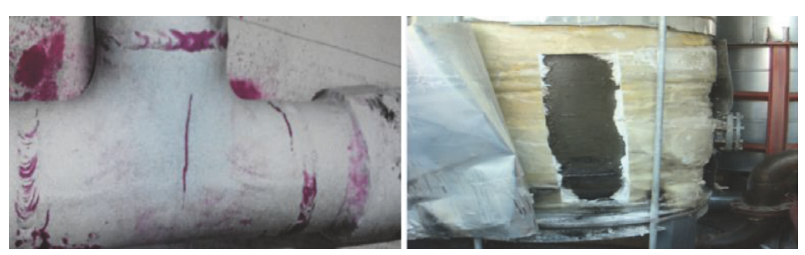

FIGURE 8: The damaged pneumatic pressure device.

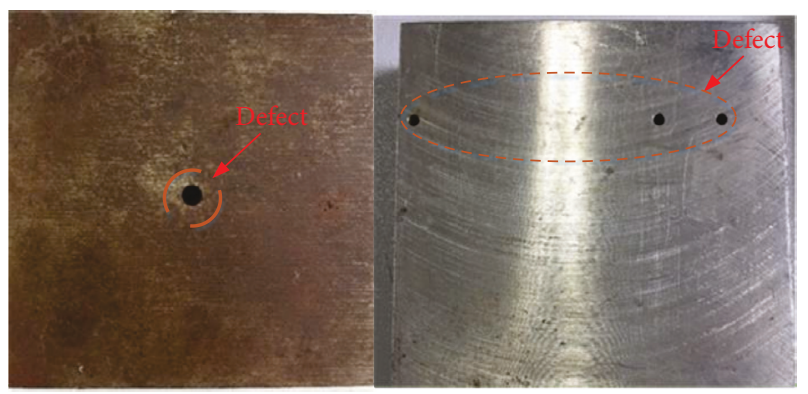

FIgURE 9: The specimen 1 and specimen 2.

physical characteristics of the defect, and the content is closer to the defect feature information. Thus the proposed algorithm is more accurate and reasonable than the ICA.

Remark 8. Step 1 realizes the segmentation of data block and the calculation of column interval and row interval. Step 2 and Figure 3 show the process of the variable interval search. Step 3 is the correlation value classification. Step 4 selects the $L$ typical TTRs based on the distance of two classes and extracts the typical features.

\section{Experimental Design}

The setup of the experiment is shown in Figure 7. It includes five functional parts: induction heater; coil; PC; timing trigger; and IR camera. The induction heater produces high frequency alternating current for coil excitation. A rectangular coil applies directional excitation to heat the sample. The timing trigger controls the time for heating the sample. The IR camera records the thermal image sequence of the sample. The analysis of experimental data is represented below.
Figure 8 shows the damaged pneumatic pressure equipment. All the test materials in this paper are provided by the China Aerodynamics Research \& Development Center, which broadly supports research on aerodynamic equipment. In view of the devices volume and degree of damage, specimen 1 and specimen 2, shown in Figure 9, are extracted from the pneumatic pressure equipment to analyze.

The defects have been detected using the method of ECPT and the algorithm presented in Section 3. The two specimens are analyzed as follows. For sample 1, the parameter index of specimen 1 is presented in Table 1 .

The process of Steps 3 and 4 is represented in Figure 10. In Step 3, set $\mathrm{p}=1, \mathrm{~b}=1, \mathrm{c}=100, \varepsilon=10^{-5}$. There are 46, 13, 35, and 2 TTRs in the corresponding parts. Through the algorithm of Step 3, in the $1_{s t}$ part, compared with other TTRs, the $2_{\text {nd }}$ possesses the maximum noncorrelation, $D I S_{2^{1}}^{i}=1.3124$. The same as in the $2_{n d}$ part, the $11_{(t h)}$ possesses the maximum noncorrelation, $D I S_{11^{2}}^{i}=1.3876$. In the $3_{r d}$ part, the $35_{t h}$ possesses the maximum noncorrelation, $D I S_{35^{3}}^{i}=1.3097$. In the $4_{t h}$, the $1_{s t}$ possesses the maximum noncorrelation, $D I S_{1^{4}}^{i}=1.3951 . T T R_{1}, T T R_{2}, T T R_{3}$, and $T T R_{4}$ have been extracted by Steps 3 and 4 . The extraction results of proposed algorithm and the ICA result are shown in Figure 11. It is obvious that the features of the selected TTRs are similar to the ICs. In Figure 12, the difference of TTRs is shown. Comparing the proposed algorithm result with the result of ICA in Figure 13, the trends of the red curves are similar to the blue curves, respectively. Moreover, Figure 14 illustrates the precise and accurate defect feature processed by the algorithm. When comparing the proposed algorithm result with the result of the ICA in Figure 15, the final result of the algorithm ignores more redundant information than the ICA result. At the same time, the defect feature extracted by the proposed algorithm is more precise and accurate than the ICA result. Consequently, the algorithm has selected the typical thermal responses and extracted main features successfully. The proposed method can not only extract the main features like the ICA, but also reduce the processed time substantially. Its biggest advantage is the efficiency. Figure 16 shows the processing time of the ICA and the proposed algorithm. It is obvious that the proposed algorithm needs less time to complete the feature extraction process. 


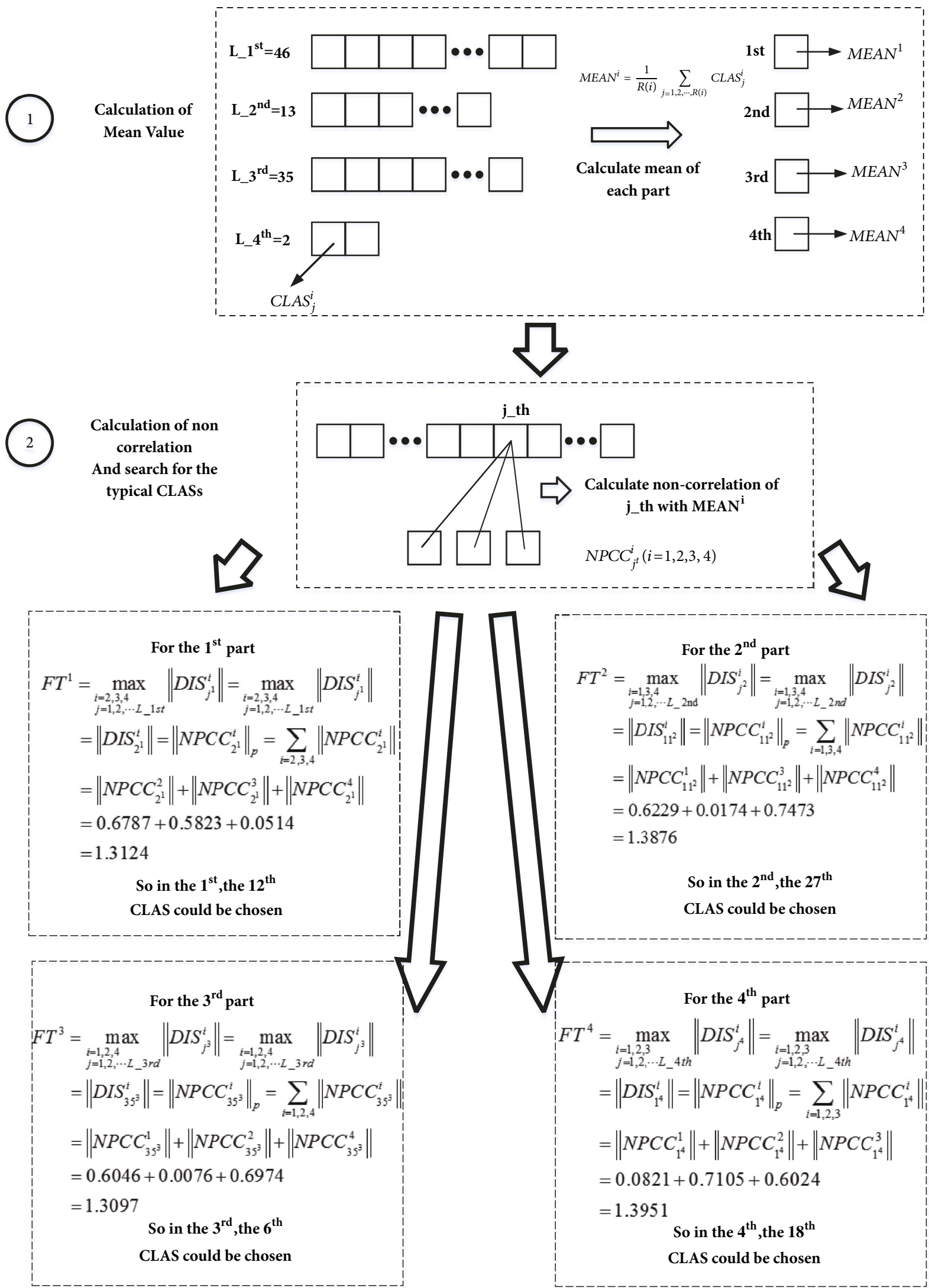

FIGURE 10: Steps 3 and 4 in proposed algorithm of sample 1. 


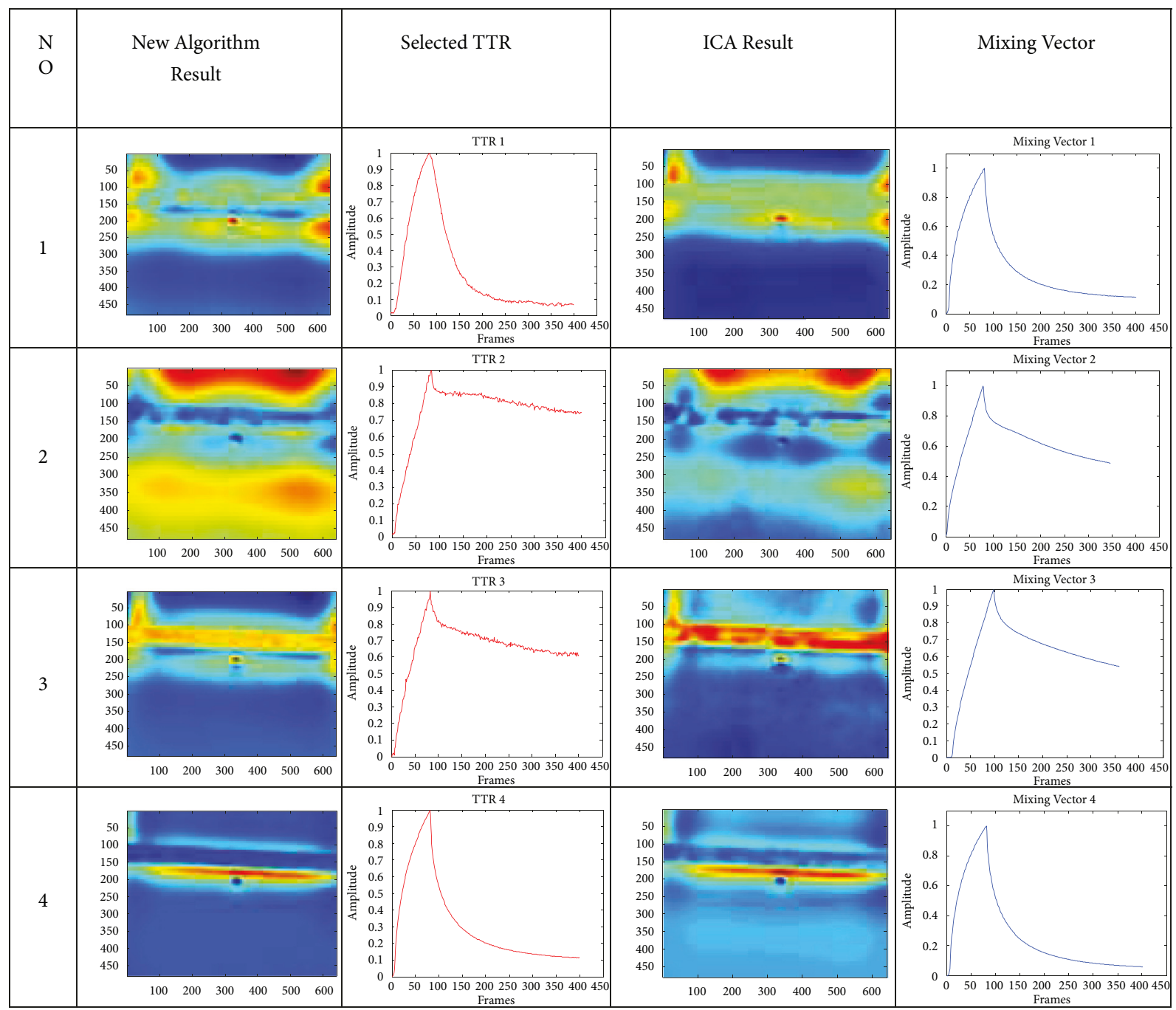

FIgURE 11: The proposed algorithm and ICA result of sample 1.

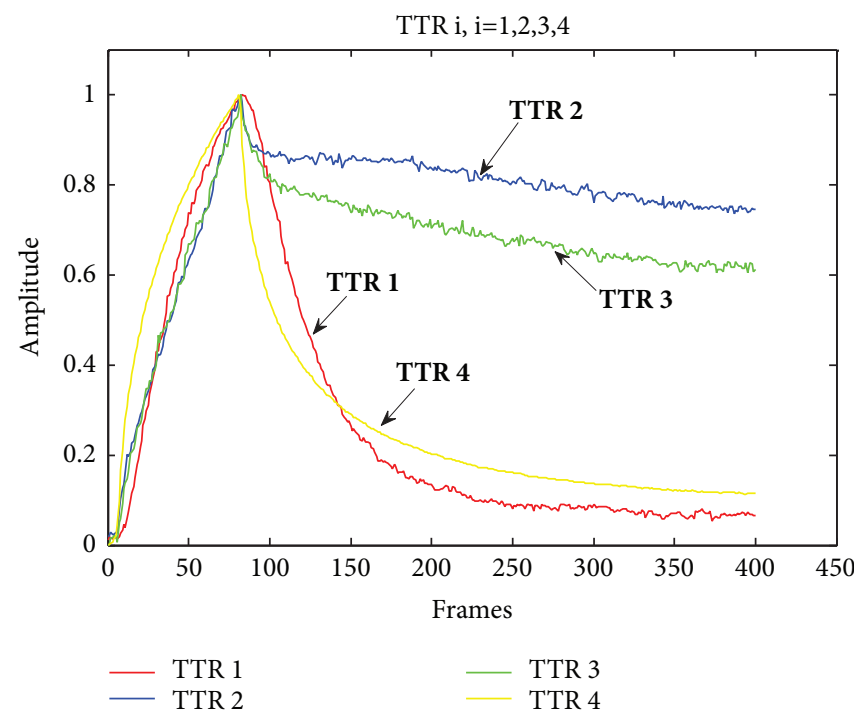

FIGURE 12: The comparison of $T T R_{1}, T T R_{2}, T T R_{3}$, and $T T R_{4}$ of sample 1 . 


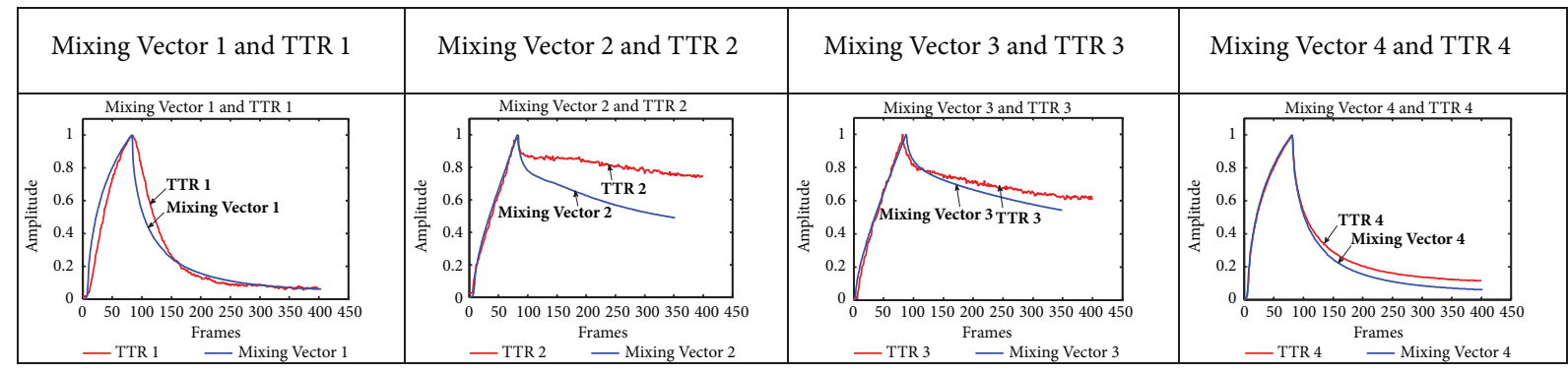

FIGURE 13: The normalized mixing vector 1 , mixing vector 2 , mixing vector 3 , mixing vector 4 , and $T T R_{1}, T T R_{2}, T T R_{3}$, and $T T R_{4}$.

TABLE 1: Parameter index of specimen 1.

\begin{tabular}{lcccc}
\hline SampleTime & TemperatureThresholds & THRE_CL & REFR & $L$ \\
\hline $17 s$ & $T(1)=40, T(2)=80$ & 0.98 & REFR $^{1}=0.98, R_{E F R^{2}=0.96, R^{2} F R^{3}=0.94}$ & 2 \\
\hline
\end{tabular}

TABle 2: Parameter index of specimen 2.

\begin{tabular}{lcccc}
\hline SampleTime & TemperatureThresholds & THRE_CL & REFR & $L$ \\
\hline $18 s$ & $T(1)=29, T(2)=40$ & 0.97 & REFR $^{1}=0.97$, REFR $^{2}=0.95$, REFR $^{3}=0.93$ & 2 \\
\hline
\end{tabular}

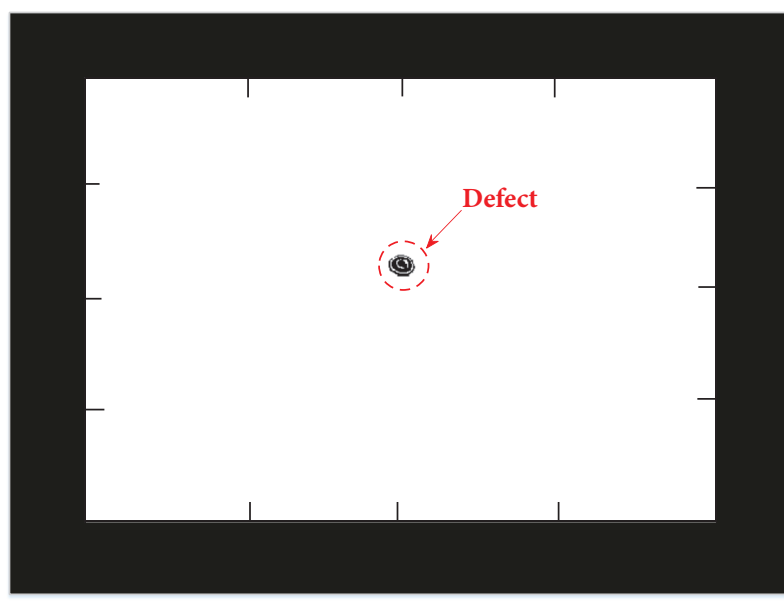

Figure 14: The defect feature of final result.

For sample 2, the parameter index of specimen 2 is presented in Table 2 .

The process of Steps 3 and 4 is represented in Figure 17. In Step 3, set $\mathrm{p}=1, \mathrm{~b}=1, \mathrm{c}=100, \varepsilon=10^{-5}$. There are $30,30,24$, and 19 TTRs in the corresponding parts. Through the algorithm of Step 3, in the $1_{s t}$ part, compared with other TTRs, the $12_{t h}$ possesses the maximum noncorrelation, $D I S_{12^{1}}^{i}=1.6693$. The same as in the $2_{n d}$ part, the $27_{t h}$ possesses the maximum noncorrelation, $D I S_{27^{2}}^{i}=1.8238$. In the $3_{r d}$ part, the $6_{t h}$ possesses the maximum noncorrelation, $D I S_{6^{3}}^{i}=1.3921$. In the $4_{t h}$, the $18_{t h}$ possesses the maximum noncorrelation, $D I S_{18^{4}}^{i}=1.1842 . T T R_{1}, T T R_{2}, T T R_{3}$, and $T T R_{4}$ have been extracted by Step 3. The extraction results of proposed algorithm and the ICA result are shown in Figure 18. The Pearson correlation coefficients between mixing vectors 1 , 2, 3, and 4 and $T T R_{1}, T T R_{2}, T T R_{3}$, and $T T R_{4}$ are 0.9786 ,
$0.9435,0.9678$, and 0.9878 , respectively. It is obvious that the features of the selected TTRs are similar to the ICs. In Figure 19, the difference of TTRs is shown. Comparing the proposed algorithm result with the result of ICA in Figure 20, the trends of the red curves are similar to the blue curves, respectively. Moreover, Figure 21 illustrates the precise and accurate defect feature processed by the algorithm. When comparing the proposed algorithm result with the result of the ICA in Figure 22, the final result of the algorithm ignores more redundant information than the ICA result. At the same time, the defect feature extracted by the proposed algorithm is more precise and accurate than the ICA result. Consequently, the algorithm has selected the typical thermal responses and extracted main features successfully. The proposed method can not only extract the main features like the ICA, but also reduce the processed time substantially. Its biggest advantage is the efficiency. Figure 23 shows the processing time of the ICA and the proposed algorithm. It is obvious that the proposed algorithm needs less time to complete the feature extraction process.

\section{Conclusion and Future Work}

In this paper, an accurate and more efficient algorithm in ECPT is proposed. The validity and efficiency of the proposed method are demonstrated with experimental results. The physical meaning of ECPT and the mathematical foundation of ICA are integrated in this proposed method. The results contribute to advancing the use of ECPT to detect defects as follows.

(1) The primary features of the thermal image sequences can be extracted by the proposed approach. Meanwhile the main features can be utilized to detect defects.

(2) Experimental results show that whitening preprocedure is time-consuming. The proposed algorithm has no data 


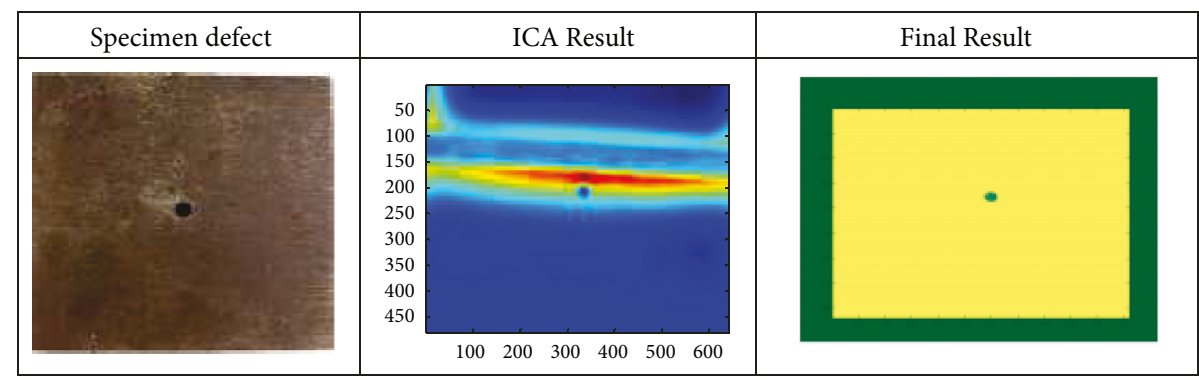

FIgURE 15: The specimen defect, the ICA result, and the final result.

TABLE 3

\begin{tabular}{|c|c|}
\hline Symbol & Notations \\
\hline$D$ & 3 dimensional matrix \\
\hline$R$ & the total number of rows in $D$ \\
\hline C & the all number of columns in $D$ \\
\hline$Z$ & the total number of the images at the taxis \\
\hline$D(r, c,:)$ & the transient thermal response \\
\hline$D\left(R_{m m}, C_{m m}, T_{m m}\right)$ & the peak temperature point $D\left(R_{m m}, C_{m m}, T_{m m}\right)=\begin{array}{c}\max \\
r=1,2 \cdots M \\
c=1,2, \cdots N \\
t=1,2, \cdots T\end{array}[D(r, c, t)]$ \\
\hline THRE_CL $L^{k}$ & the temperature thresholds in searching for the variable interval $C L^{k}$ \\
\hline$T H R E \_R L^{p}$ & the temperature thresholds in searching for the variable interval $R L^{p}$ \\
\hline $\operatorname{len}_{k}$ & the number of each block in searching for the variable interval $C L^{k}$ \\
\hline $\operatorname{len}_{p}$ & the number of each block in searching for the variable interval $R L^{p}$ \\
\hline$\left(D_{k}^{p}(r, c,:)\right.$ & the transient thermal response in $k$ part of row and p part of column \\
\hline $2 \leq L \leq g$ & the final number of classifications in Step 2 \\
\hline $\operatorname{PCC}(X, Y)$ & the computation of $\operatorname{PCC}(X, Y)=(1 /(n-1)) \sum_{i=1}^{n}\left(\left(X_{i}-\bar{X}\right) / \sigma_{X}\right)\left(\left(Y_{i}-\bar{Y}\right) / \sigma_{Y}\right)$ \\
\hline$C L A S_{j}^{i}$ & the $j^{\text {th }}$ TTR of $i^{\text {th }}$ class \\
\hline$j^{t}$ & the $j^{\text {th }}$ TTR in $t^{\text {th }}$ classification \\
\hline
\end{tabular}

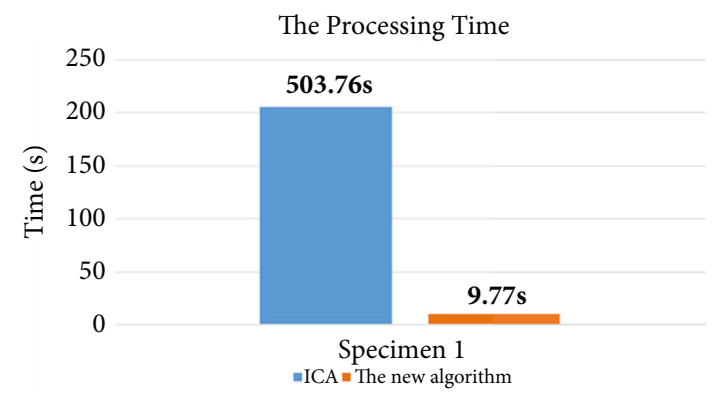

FIGURE 16: The time comparison for specimen 1. less than that of in ICA. Hence, in the postprocessing, the proposed algorithm avoids redundancy calculation and possesses higher efficiency than the ICA. So the proposed algorithm is more accurate and reasonable than the ICA.

Future work will pay more attention to how to enhance the efficiency and accuracy of the novel algorithm. In the experiment, some external interference will bring plenty of noise to influence the precision. Moreover, the change of threshold values in the algorithm is also anticipated to impact the efficiency of the detection. Therefore, it is worth researching how to reduce the external interference and to adjust the threshold value.

\section{Appendix}

whitening procedure. So the proposed algorithm is more effective.

(3) After processing of pretreatment and whitening, the amount of data in the proposed algorithm is much

\section{Notations of the Proposed Algorithm}

To make the algorithm explicit and clear, the mathematics definitions in the algorithm are shown in Table 3. 


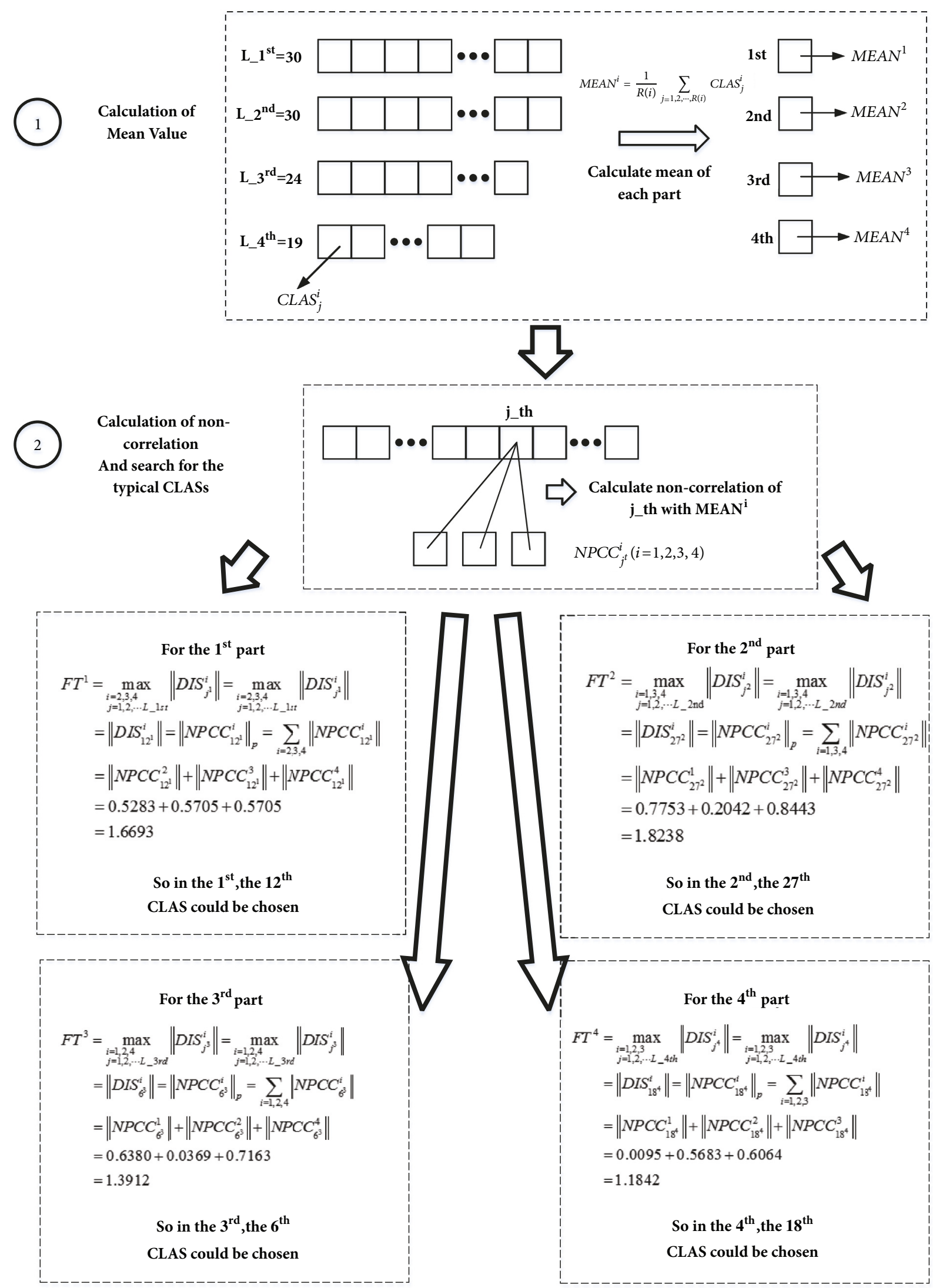

FIGURE 17: Steps 3 and 4 in proposed algorithm of sample 2. 


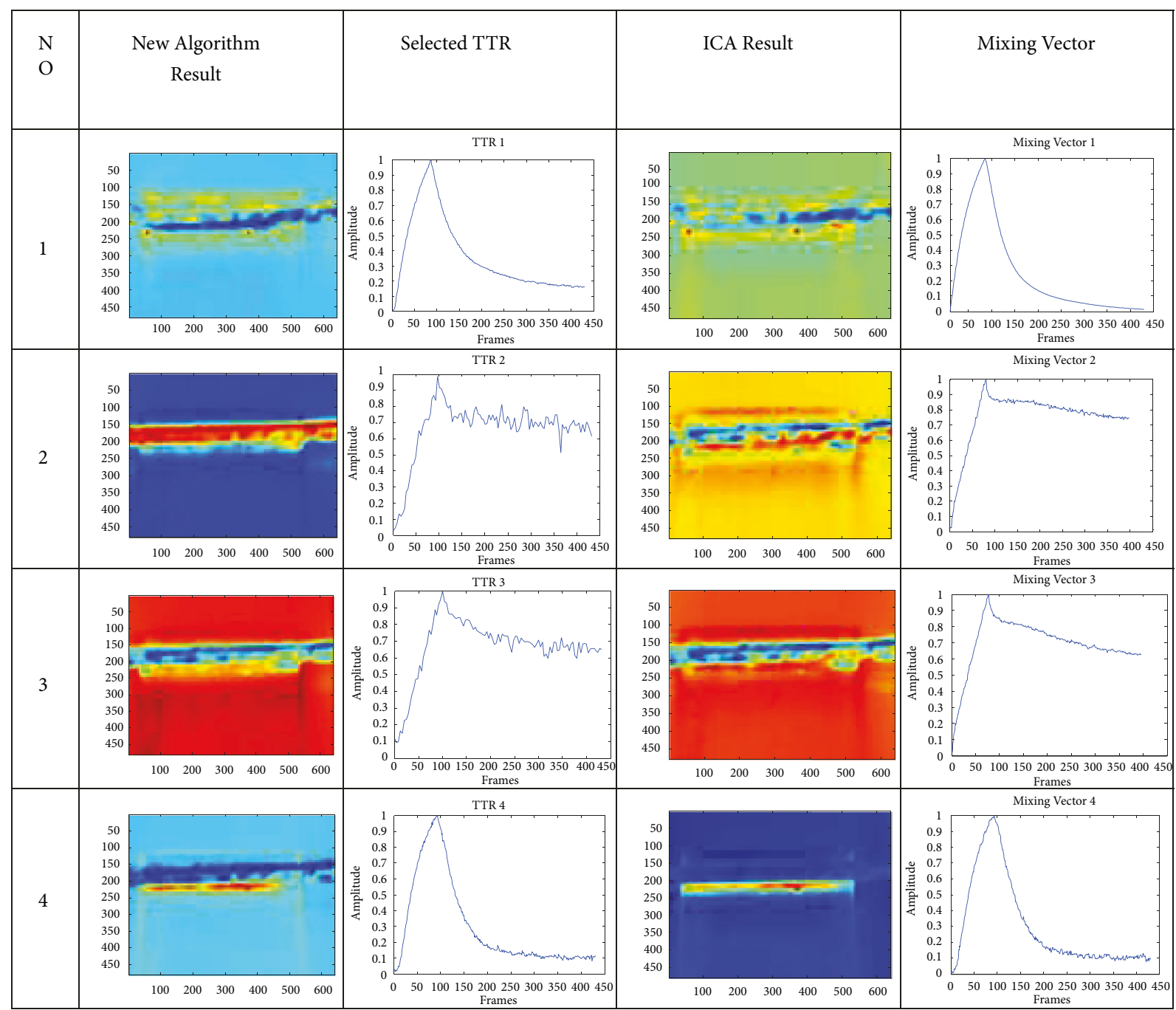

FIGURE 18: The proposed algorithm and ICA result of sample 2.

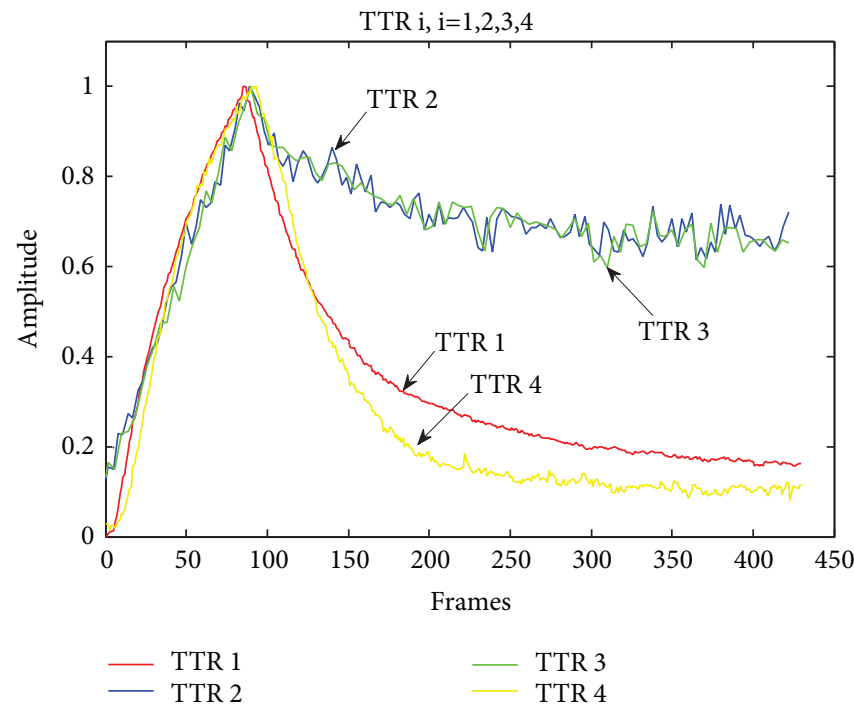

FIgURE 19: The comparison of $T T R_{1}, T T R_{2}, T T R_{3}$, and $T T R_{4}$ of sample 2. 


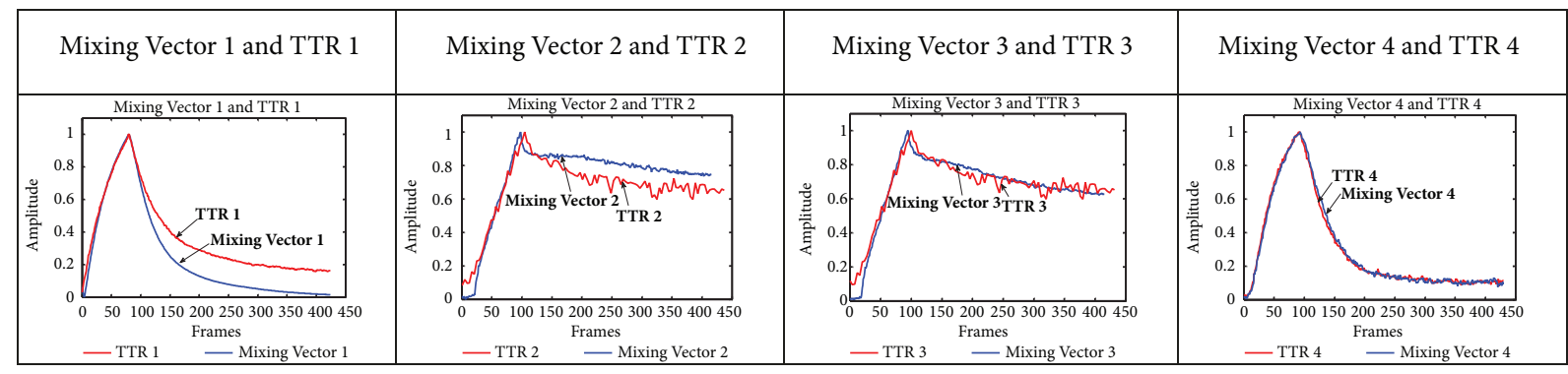

FIGURE 20: The normalized mixing vector 1 , mixing vector 2 , mixing vector 3 , mixing vector 4 , and $T T R_{1}, T T R_{2}, T T R_{3}$, and $T T R_{4}$.

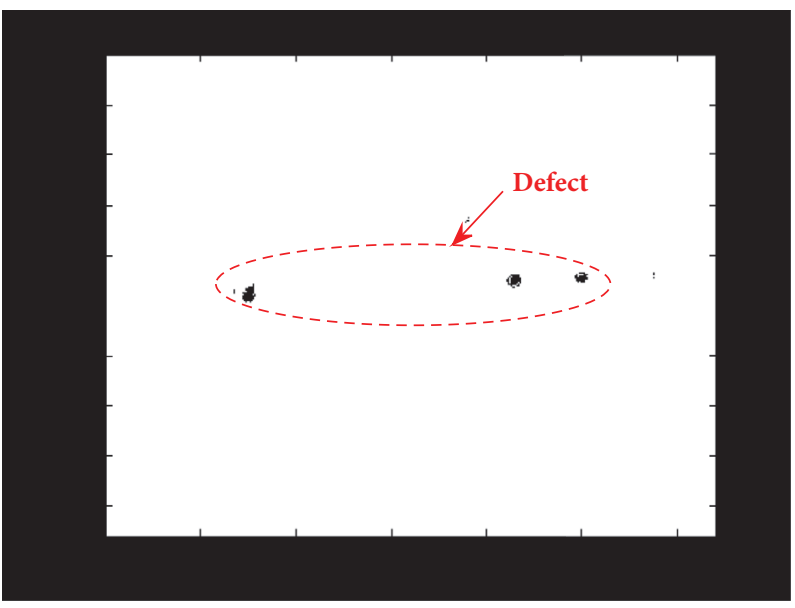

FIGURE 21: The defect feature of final result.

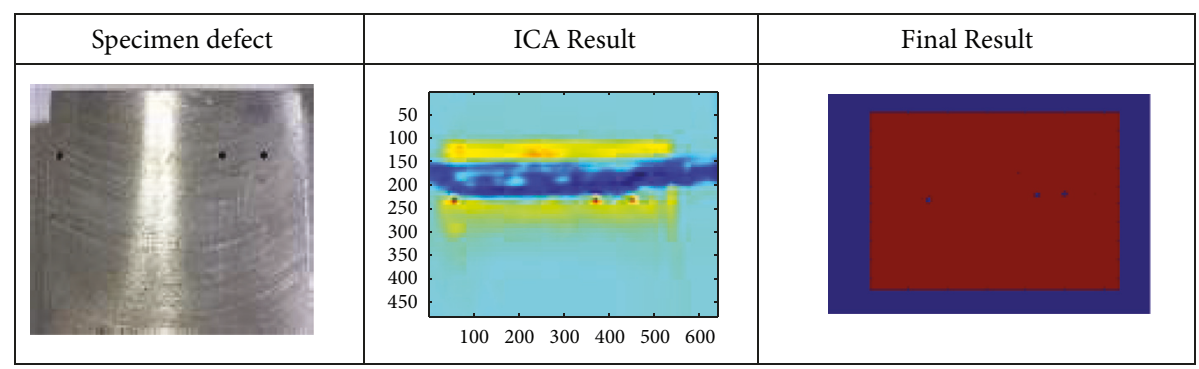

FIGURE 22: The specimen defect, the ICA result, and the final result.

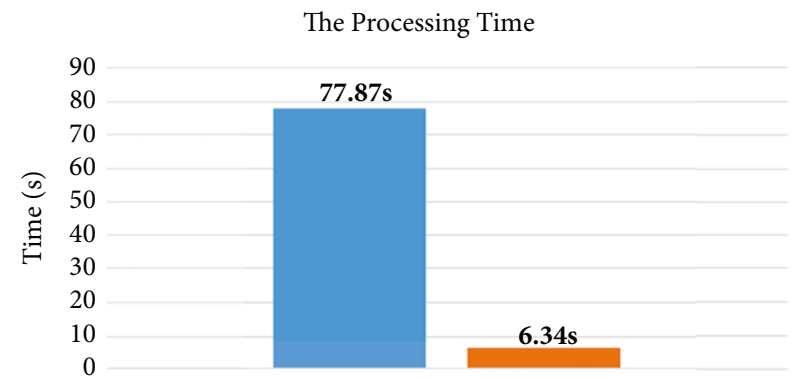

Specimen 1

$=\mathrm{ICA} \equiv$ The new algorithm

FIGURE 23: The time comparison for specimen 2. 


\section{Data Availability}

The data used to support the findings of this study are available from the corresponding author upon request.

\section{Conflicts of Interest}

The authors declare that they have no conflicts of interest.

\section{Acknowledgments}

This work was supported by National Basic Research Program of China (Grants nos. 61873305, 61671109, U1830207, 51502338, and 61503064), 2018JY0410, Open Foundation of Hypervelocity Impact 20181102, and the Fundamental Research Funds for the Central Universities.

\section{References}

[1] J. Lee, Y. Choi, C. Jo, and D. Chang, "Design of a prismatic pressure vessel: An engineering solution for non-stiffened-type vessels," Ocean Engineering, vol. 142, pp. 639-649, 2017.

[2] H. Al-Gahtani, A. Khathlan, M. Sunar, and M. Naffa'a, "Local pressure testing of spherical vessels," International Journal of Pressure Vessels and Piping, vol. 114-115, pp. 61-68, 2014.

[3] J. J. Proczka, K. Muralidharan, D. Villela, J. H. Simmons, and G. Frantziskonis, "Guidelines for the pressure and efficient sizing of pressure vessels for compressed air energy storage," Energy Conversion and Management, vol. 65, pp. 597-605, 2013.

[4] P. Blanc-Vannet, "Burst pressure reduction of various thermoset composite pressure vessels after impact on the cylindrical part," Composite Structures, vol. 160, pp. 706-711, 2017.

[5] X. Maldague, Theory and Practice of Infrared Technology for Nondestructive Testing, John Wiley \& Sons, 2001.

[6] S. Marinetti, E. Grinzato, P. G. Bison et al., "Statistical analysis of IR thermographic sequences by PCA," Infrared Physics \& Technology, vol. 46, no. 1-2, pp. 85-91, 2004.

[7] Y. Cheng, L. Tian, C. Yin, X. Huang, and L. Bai, "A magnetic domain spots filtering method with self-adapting threshold value selecting for crack detection based on the MOI," Nonlinear Dynamics, vol. 86, no. 2, pp. 741-750, 2016.

[8] X. Huang, C. Yin, J. Huang et al., "Hypervelocity impact of TiB2-based composites as front bumpers for space shield applications," Materials and Corrosion, vol. 97, pp. 473-482, 2016.

[9] X. Xie, D. Yue, H. Zhang, and Y. Xue, "Fault Estimation Observer Design for Discrete-Time Takagi-Sugeno Fuzzy Systems Based on Homogenous Polynomially Parameter-Dependent Lyapunov Functions," IEEE Transactions on Cybernetics, vol. 47, no. 9, pp. 2504-2513, 2017.

[10] X. Huang, C. Yin, S. Dadras, Y. Cheng, and L. Bai, "Adaptive rapid defect identification in ECPT based on K-means and automatic segmentation algorithm," Journal of Ambient Intelligence and Humanized Computing, pp. 1-18, 2017.

[11] K. Schabowicz and T. Gorzelańczyk, "A nondestructive methodology for the testing of fibre cement boards by means of a non-contact ultrasound scanner," Construction and Building Materials, vol. 102, pp. 200-207, 2016.

[12] A. Salazar, L. Vergar, and R. Llinares, "Learning material defect patterns by separating mixtures of independent component analyzers from NDT sonic signals," Mechanical Systems and Signal Processing, vol. 24, no. 6, pp. 1870-1886, 2010.
[13] C.-L. Lim, R. Paramesran, W. Jassim, Y.-P. Yu, and K. N. Ngan, "Blind image quality assessment for Gaussian blur images using exact Zernike moments and gradient magnitude," Journal of The Franklin Institute, vol. 353, no. 17, pp. 4715-4733, 2016.

[14] D. Liu, C. Wu, Q. Zhou, and H.-K. Lam, "Fuzzy guaranteed cost output tracking control for fuzzy discrete-time systems with different premise variables," Complexity, vol. 21, no. 5, pp. 265276, 2016.

[15] P. Liu and F. Teng, "Multiple criteria decision making method based on normal interval-valued intuitionistic fuzzy generalized aggregation operator," Complexity, vol. 21, no. 5, pp. 277290, 2016.

[16] P. Zhu, C. Yin, Y. Cheng et al., "An improved feature extraction algorithm for automatic defect identification based on eddy current pulsed thermography," Mechanical Systems \& Signal Processing, vol. 113, pp. 5-21, 2018.

[17] H. Yu, X. Xie, J. Zhang, D. Ning, and Y.-W. Jing, "Relaxed fuzzy observer-based output feedback control synthesis of discretetime nonlinear control systems," Complexity, vol. 21, no. S1, pp. 593-601, 2016.

[18] J. Cao, K. Zhang, M. Luo, C. Yin, and X. Lai, "Extreme learning machine and adaptive sparse representation for image classification," Neural Networks, vol. 81, pp. 91-102, 2016.

[19] C. Yin, X. Huang, Y. Chen, S. Dadras, S.-M. Zhong, and Y. Cheng, "Fractional-order exponential switching technique to enhance sliding mode control," Applied Mathematical Modelling: Simulation and Computation for Engineering and Environmental Systems, vol. 44, pp. 705-726, 2017.

[20] C. Yin, S. Dadras, X. Huang, J. Mei, H. Malek, and Y. Cheng, "Energy-saving control strategy for lighting system based on multivariate extremum seeking with Newton algorithm," Energy Conversion and Management, vol. 142, pp. 504-522, 2017.

[21] Y. Song, Z. Wang, S. Liu, and G. Wei, "N-step MPC with persistent bounded disturbances under stochastic communication protocol," IEEE Transactions on Systems, Man, and Cybernetics: Systems, pp. 1-11, 2018. 


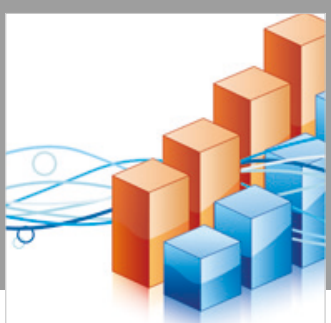

Advances in

Operations Research

\section{-n-m}
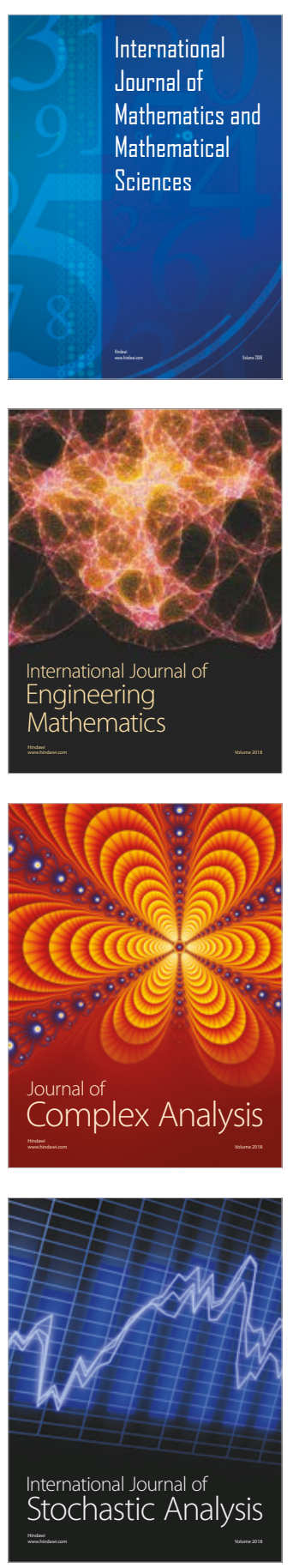
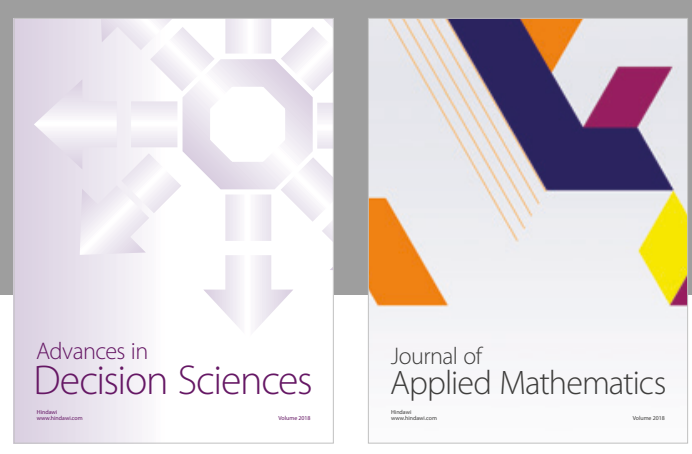

Journal of

Applied Mathematics
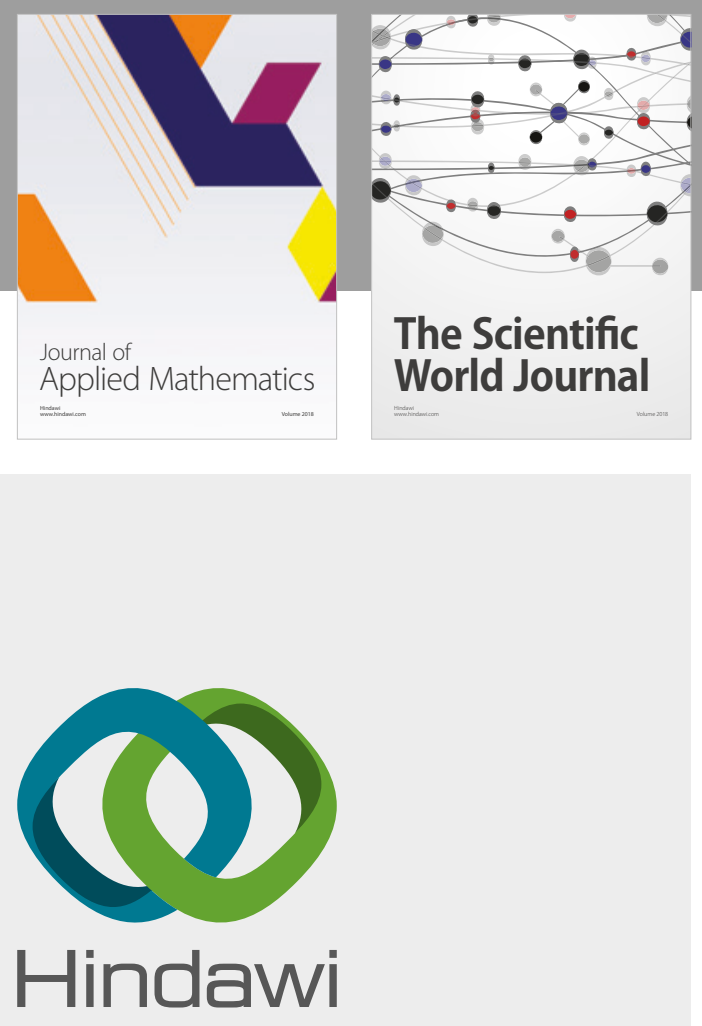

Submit your manuscripts at

www.hindawi.com

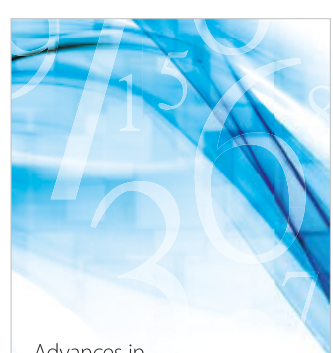

Advances in
Numerical Analysis
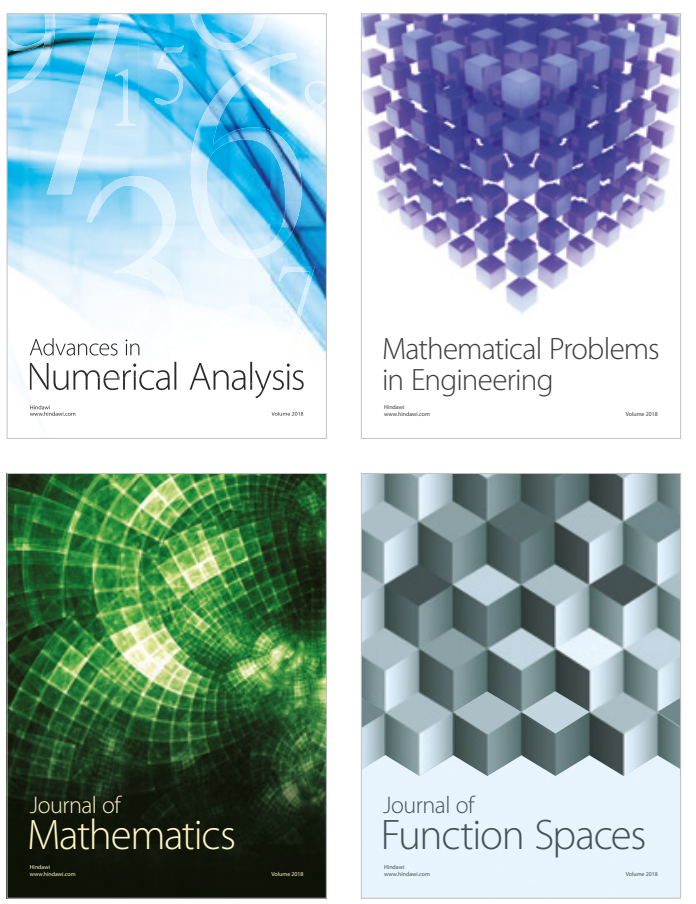

Mathematical Problems in Engineering

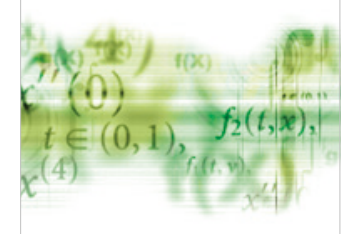

International Journal of

Differential Equations

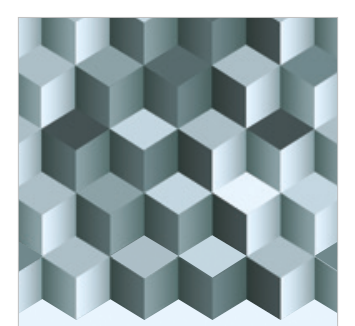

Journal of

Function Spaces
The Scientific

World Journal

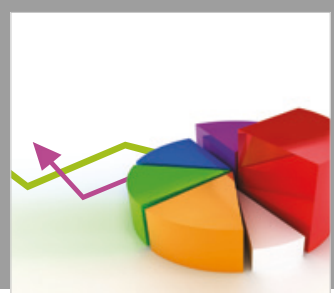

Journal of

Probability and Statistics
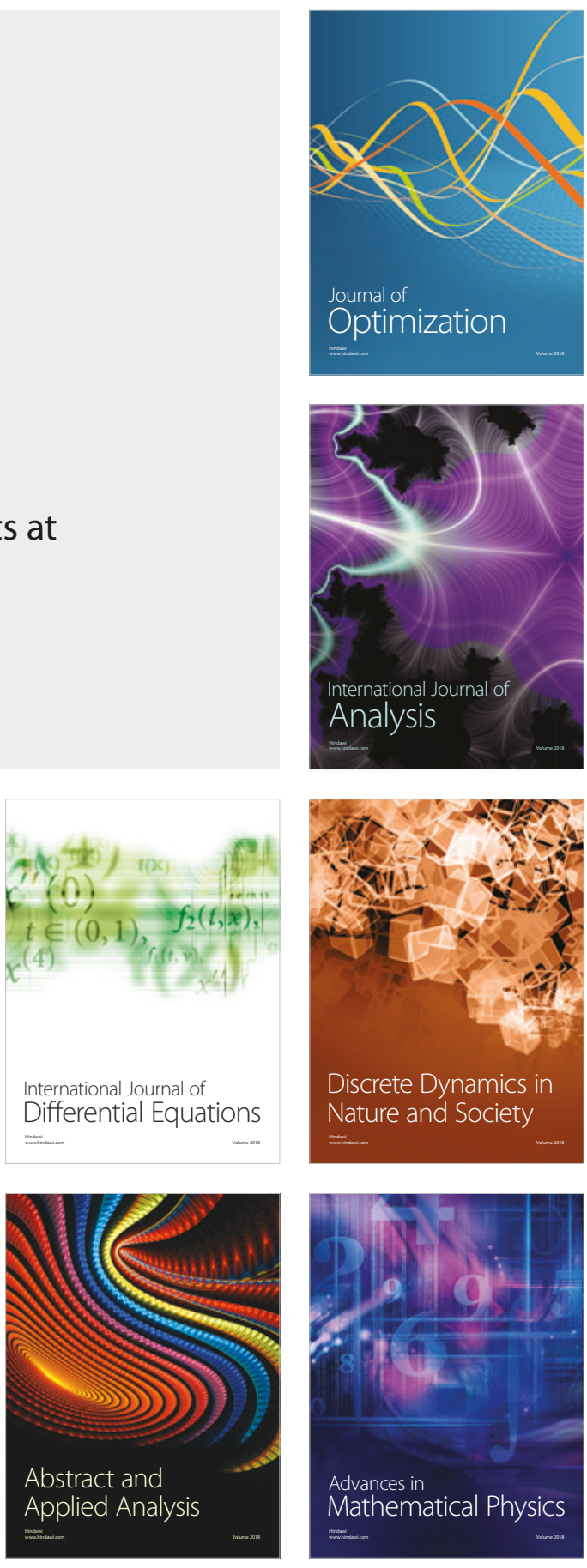